\title{
NUSTAR OBSERVATIONS OF THE COMPTON-THICK ACTIVE GALACTIC NUCLEUS AND ULTRALUMINOUS X-RAY SOURCE CANDIDATE IN NGC 5643
}

\author{
A. Annuar ${ }^{1}$, P. Gandhi ${ }^{1,2}$, D. M. Alexander ${ }^{1}$, G. B. Lansbury ${ }^{1}$, P. Arévalo ${ }^{3,4}$, D. R. Ballantyne ${ }^{5}$, M. Baloković ${ }^{6}$, \\ F. E. Bauer ${ }^{4,7,8,9}$, S. E. Boggs ${ }^{10}$, W. N. Brandt ${ }^{11,12,13}$, M. Brightman ${ }^{6}$, F. E. Christensen ${ }^{14}$, W. W. Craig ${ }^{10,15}$, A. Del Moro ${ }^{1}$, \\ C. J. Hailey ${ }^{16}$, F. A. Harrison ${ }^{6}$, R. C. Hickox ${ }^{17}$, G. Matt ${ }^{18}$, S. Puccetti ${ }^{19,20}$, C. Ricci ${ }^{21}$, J. R. Rigby ${ }^{22}$, D. Stern ${ }^{23}$, \\ D. J. WALton ${ }^{6,23}$, L. ZapPaCOSTA ${ }^{20}$, AND W. Zhang ${ }^{22}$ \\ ${ }^{1}$ Centre for Extragalactic Astronomy, Department of Physics, University of Durham, South Road, Durham, DH1 3LE, UK \\ ${ }^{2}$ Department of Physics and Astronomy, Faculty of Physical Sciences and Engineering, University of Southampton, Southampton, SO17 1BJ, UK \\ ${ }^{3}$ Instituto de Física y Astronomía, Facultad de Ciencias, Universidad de Valparaíso, Gran Bretana N 1111, Playa Ancha, Valparaíso, Chile \\ ${ }^{4}$ EMBIGGEN Anillo, Concepción, Chile \\ ${ }^{5}$ Center for Relativistic Astrophysics, School of Physics, Georgia Institute of Technology, Atlanta, GA 30332, USA \\ ${ }^{6}$ Cahill Center for Astronomy and Astrophysics, California Institute of Technology, Pasadena, CA 91125, USA \\ 7 Pontificia Universidad Católica de Chile, Instituto de Astrofísica, Casilla 306, Santiago 22, Chile \\ ${ }^{8}$ Millenium Institute of Astrophysics, Santiago, Chile \\ ${ }_{10}^{9}$ Space Science Institute, 4750 Walnut Street, Suite 205, Boulder, CO 80301, USA \\ ${ }_{10}$ Space Sciences Laboratory, University of California, Berkeley, CA 94720, USA \\ ${ }^{11}$ Department of Astronomy and Astrophysics, The Pennsylvania State University, 525 Davey Lab, University Park, PA 16802, USA \\ ${ }_{12}$ Institute for Gravitation and the Cosmos, The Pennsylvania State University, University Park, PA 16802, USA \\ ${ }^{13}$ Department of Physics, The Pennsylvania State University, 525 Davey Lab, University Park, PA 16802, USA \\ ${ }^{14}$ DTU Space, National Space Institute, Technical University of Denmark, Elektrovej 327, DK-2800 Lyngby, Denmark \\ ${ }^{15}$ Lawrence Livermore National Laboratory, Livermore, CA 94550, USA \\ ${ }^{16}$ Columbia Astrophysics Laboratory, Columbia University, New York, NY 10027, USA \\ ${ }^{17}$ Department of Physics and Astronomy, Dartmouth College, 6127 Wilder Laboratory, Hanover, NH 03755, USA \\ ${ }^{18}$ Dipartimento di Matematica e Fisica, Universitá degli Studi Roma Tre, via della Vasca Navale 84, I-00146 Roma, Italy \\ ${ }^{19}$ ASI Science Data Center, via Galileo Galilei, I-00044 Frascati, Italy \\ ${ }^{20}$ Observatorio Astronomico di Roma (INAF), via Frascati 33, I-00040 Monte Porzio Catone (Roma), Italy \\ ${ }^{21}$ Department of Astronomy, Kyoto University, Kitashirakawa-Oiwake-cho, Sakyo-ku, Kyoto 606-8502, Japan \\ ${ }^{22}$ NASA Goddard Space Flight Center, Greenbelt, MD 20771, USA \\ ${ }^{23}$ Jet Propulsion Laboratory, California Institute of Technology, Pasadena, CA 91109, USA \\ Received 2015 June 2; accepted 2015 October 4; published 2015 December 4
}

\begin{abstract}
We present two Nuclear Spectroscopic Telescope Array (NuSTAR) observations of the local Seyfert 2 active galactic nucleus (AGN) and an ultraluminous X-ray source (ULX) candidate in NGC 5643. Together with archival data from Chandra, XMM-Newton, and Swift-BAT, we perform a high-quality broadband spectral analysis of the AGN over two decades in energy $(\sim 0.5-100 \mathrm{keV})$. Previous X-ray observations suggested that the AGN is obscured by a Compton-thick (CT) column of obscuring gas along our line of sight. However, the lack of highquality $\gtrsim 10 \mathrm{keV}$ observations, together with the presence of a nearby X-ray luminous source, NGC $5643 \mathrm{X}-1$, have left significant uncertainties in the characterization of the nuclear spectrum. NuSTAR now enables the AGN and NGC $5643 \mathrm{X}-1$ to be separately resolved above $10 \mathrm{keV}$ for the first time and allows a direct measurement of the absorbing column density toward the nucleus. The new data show that the nucleus is indeed obscured by a CT column of $N_{\mathrm{H}} \gtrsim 5 \times 10^{24} \mathrm{~cm}^{-2}$. The range of $2-10 \mathrm{keV}$ absorption-corrected luminosity inferred from the bestfitting models is $L_{2-10, \text { int }}=(0.8-1.7) \times 10^{42} \mathrm{erg} \mathrm{s}^{-1}$, consistent with that predicted from multiwavelength intrinsic luminosity indicators. In addition, we also study the NuSTAR data for NGC $5643 \mathrm{X}-1$ and show that it exhibits evidence of a spectral cutoff at energy $E \sim 10 \mathrm{keV}$, similar to that seen in other ULXs observed by NuSTAR. Along with the evidence for significant X-ray luminosity variations in the $3-8 \mathrm{keV}$ band from 2003 to 2014 , our results further strengthen the ULX classification of NGC $5643 \mathrm{X}-1$.
\end{abstract}

Key words: galaxies: active - galaxies: nuclei - techniques: spectroscopic - X-rays: galaxies - X-rays: individual (NGC 5643, NGC 5643 X-1)

\section{INTRODUCTION}

Compton-thick active galactic nuclei (CTAGNs) are expected to constitute a significant fraction of the overall AGN population in the local universe, accounting for $\sim 20 \%$ $30 \%$ of AGNs according to multiwavelength studies (e.g., Risaliti et al. 1999; Burlon et al. 2011; Goulding et al. 2011). Many studies also predict that CTAGNs provide a substantial contribution to the cosmic X-ray background, responsible for $10 \%-25 \%$ of the flux at the peak energy, $\sim 30 \mathrm{keV}$ (e.g., Gilli et al. 2007; Treister et al. 2009; Draper \& Ballantyne 2010; Akylas et al. 2012; Ueda et al. 2014). Yet, their census is still far from complete. The high line of sight column density in CTAGNs $\left(N_{\mathrm{H}} \geqslant 1 / \sigma_{\mathrm{T}}=1.5 \times 10^{24} \mathrm{~cm}^{-2}\right.$, where $\sigma_{\mathrm{T}}$ is the Thomson scattering constant), generally attributed to the parsec-scale circumnuclear torus of AGN unification schemes, as well as larger-scale molecular clouds and dust lanes, results in severe attenuation of the direct X-ray emission from CTAGNs at energies below $10 \mathrm{keV}$. This is why observations at higher energies are needed to probe this direct component and provide unambiguous identification of CTAGNs. However, even at $E>10 \mathrm{keV}$, the remaining flux that we observe in the most extreme CTAGN will be from photons scattered or reflected from the backside of the torus, and will comprise just 
a few percent of the intrinsic AGN power (e.g., Iwasawa et al. 1997; Matt et al. 2000; Baloković et al. 2014). This makes the identification and characterization of CTAGNs a challenging task.

To date, only $\sim 20$ AGNs within $\approx 200 \mathrm{Mpc}$ have been confirmed as CT based upon detailed X-ray spectral characterization (Della Ceca et al. 2008; Goulding et al. 2012; Gandhi et al. 2014). This corresponds to a fraction of $\ll 1 \%$ of the total AGN population expected within that volume, suggesting that the vast majority of CTAGNs are yet to be found even in the local universe. These bona fide CTAGNs were unambiguously identified based upon a detection at energies above $10 \mathrm{keV}$ and the presence of a $\mathrm{Fe} \mathrm{K} \alpha$ line at $6.4 \mathrm{keV}$ with high equivalent width, $\mathrm{EW} \gtrsim 1 \mathrm{keV}$. The identification and characterization of all the CTAGNs is important in order to form an accurate census of accretion in the local universe, since much of the growth of supermassive black holes is thought to occur in such heavily obscured phases (e.g., Fabian 1999; Alexander \& Hickox 2012). Therefore, an accurate local benchmark is important for extrapolating the results to higher redshifts.

We have started a program to study a complete, volumelimited $(D<15 \mathrm{Mpc})$, mid-infrared (MIR) selected AGN sample from Goulding \& Alexander (2009), with the main goal of constraining the population of CTAGNs and the $N_{\mathrm{H}}$ distribution of AGNs in the local universe. CTAGN candidates from the sample were identified using multiwavelength selections, such as X-ray spectroscopy, and intrinsic $2-10 \mathrm{keV}$ luminosity indicators from high spatial resolution $12 \mu \mathrm{m}$ continua and [O $\mathrm{III}] \lambda 5007 \AA$ line luminosities corrected for the Balmer decrement. One of the candidates that stands out in the sample as being CT based on these analyses is NGC 5643. NGC 5643 is a nearby face-on $\left(i \approx 30^{\circ}\right)^{24} \mathrm{SAB}(\mathrm{rs}) \mathrm{C}$ galaxy hosting a low-luminosity Seyfert 2 nucleus (Phillips et al. 1983). It has a redshift of $z=0.0040$, corresponding to a metric/proper distance of $D=13.9 \mathrm{Mpc}$ under the assumption of the Mould et al. (2000) cosmic attractor model (Sanders et al. 2003). ${ }^{25}$

NGC 5643 features a compact radio core with two-sided, kiloparsec-scale lobes in an east-west orientation (Morris et al. 1985), and a cospatial one-sided $\mathrm{H} \alpha$ and [O III] emission line region extending eastward of the nucleus for at least $1.8 \mathrm{kpc}$ (Simpson et al. 1997). Despite the intense star formation episodes occuring in the spiral arm, MIR diagnostics suggest that the AGN still dominates the overall IR (8-1000 $\mu \mathrm{m}$ ) energy budget (Genzel et al. 1998). Comparisons of optical spectra with synthesis models, however, are consistent with a "starburst/Seyfert 2 composite" spectrum (Cid Fernandes et al. 2001). Using $\mathrm{Br} \gamma$ emission, Davies et al. (2014) found no ongoing star formation activity in the nucleus, although the possibility of a recent (terminated) starburst cannot be excluded. This source also shows spatially resolved molecular gas flowing out from the AGN at a rate of $10 M_{\odot}$ $\mathrm{yr}^{-1}$ (Davies et al. 2014). Although water maser emission associated with the AGN has been detected in NGC 5643, a corresponding spatially resolved map, which would allow for a direct measurement of the supermassive black hole mass $\left(M_{\mathrm{BH}}\right)$, is not yet available (Greenhill et al. 2003). However, an

\footnotetext{
${ }^{24}$ The host galaxy inclination was obtained from the HyperLeda website (http://leda.univ-lyon1.fr/)

25 Mould et al. (2000) adjusts heliocentric redshifts to the centroid of the Local Group, taking into account the gravitational attraction toward the Virgo Cluster, the Great Attractor, and the Shapley supercluster.
}

indirect $M_{\mathrm{BH}}$ measurement from the galaxy stellar velocity dispersion $\left(\sigma_{*}\right)$ provides an estimated black hole mass of $M_{\mathrm{BH}}=10^{6.4} M_{\odot}\left(\right.$ Goulding et al. 2010). ${ }^{26}$

In X-rays, NGC 5643 has been observed by ASCA (Guainazzi et al. 2004), BeppoSAX (Maiolino et al. 1998), ROSAT (Guainazzi et al. 2004), Chandra (Bianchi et al. 2006), and XMM-Newton (Guainazzi et al. 2004; Matt et al. 2013). Dramatic spectral changes were observed between the XMMNewton observation carried out in 2003, and the ASCA and BeppoSAX observations performed earlier. However, the pointspread function (PSF) of ASCA and BeppoSAX were not sufficient to separate the emission of the nucleus from that of a nearby X-ray source (at an angular separation of $\sim 50^{\prime \prime}$ ), NGC $5643 \mathrm{X}-1$, which was found to be very bright at the time of the XMM-Newton observation (Guainazzi et al. 2004; hereafter G04). Therefore, it remained unclear which source was responsible for the spectral variability observed. Comparisons of the XMM-Newton observations in 2003 and 2009 showed that there is no significant variation in the spectrum of the AGN (Matt et al. 2013; hereafter M13). However, the off-nuclear source was found to be more than a factor of two fainter in flux in 2009 (M13) than in 2003 (G04).

The Chandra image of the AGN shows that the soft X-ray emission $(E \lesssim 2 \mathrm{keV})$ of the nucleus is spatially correlated with the [O III] emission (Bianchi et al. 2006), consistent with what is commonly observed for many Seyfert 2 galaxies. The dominant power source of this soft X-ray emission appears to be photoionization from the AGN, although it is still unclear how much collisional ionization contributes to the overall $\mathrm{X}$-ray emission (M13). Above $2 \mathrm{keV}$, the X-ray spectra from these various observations show indications of the nucleus being absorbed by CT material. The evidence for this is the detection of a prominent $\mathrm{Fe} \mathrm{K} \alpha$ line $(\mathrm{EW}>1 \mathrm{keV})$ and a flat photon index below $10 \mathrm{keV}\left(\Gamma_{2-10}<1\right)$, which are characteristics of a reflection-dominated spectrum. Analysis of the low signal-to-noise ratio $(\mathrm{S} / \mathrm{N})$ BeppoSAX spectrum where NGC 5643 was detected only up to $10 \mathrm{keV}$, combined with an upper limit for the $15-100 \mathrm{keV}$ band, suggested a tentative lower limit to the column density of $N_{\mathrm{H}}>10^{25} \mathrm{~cm}^{-2}$ (Maiolino et al. 1998).

The true nature of the off-nuclear X-ray source, NGC 5643 $\mathrm{X}-1$, is still uncertain. It is located in the outskirts of the host galaxy optical emission and identification of counterparts at other wavelengths has been ambiguous (G04). It is highly likely that the source is located inside the galaxy and therefore would be a powerful ultraluminous X-ray source (ULX) with $L_{2-10} \approx 1.7 \times 10^{40} \mathrm{erg} \mathrm{s}^{-1}$ based on the flux observed in 2003 (G04). This is comparable to the observed luminosity of the AGN itself; i.e., $L_{2-10} \approx 1.9 \times 10^{40} \mathrm{erg} \mathrm{s}^{-1}$ (G04).

In this paper, we present new Nuclear Spectroscopic Telescope Array (NuSTAR) observations of NGC 5643 in which the AGN and the off-nuclear source, NGC 5643 X-1, are clearly resolved and detected at hard X-ray energies $(>10 \mathrm{keV})$ for the first time. This allows us to provide the most accurate spectral analysis of the AGN to date. The aim of this paper is to characterize the broadband spectrum of the AGN by combining our NuSTAR data with existing data from Chandra, $X M M-N e w t o n$, and Swift-BAT. We also present the NuSTAR

\footnotetext{
26 The same $M_{\mathrm{BH}}$ estimate is obtained using an updated value of $\sigma_{*}$ derived from the $[\mathrm{O}$ III] $\lambda 5007 \AA$ emission line width from Gu et al. (2006) and the latest $M_{\mathrm{BH}}-\sigma_{*}$ correlation by McConnell \& Ma (2013).
} 
Table 1

X-Ray Observations Log of NGC 5643

\begin{tabular}{|c|c|c|c|c|c|}
\hline (1) & (2) & (3) & $\begin{array}{c}\text { Energy Band } \\
(\mathrm{keV}) \\
(4)\end{array}$ & $\begin{array}{c}\text { Net Exposure Time } \\
\text { (ks) } \\
(5)\end{array}$ & $\begin{array}{c}\text { Net Count Rate } \\
\left(10^{-2} \text { cts s}{ }^{-1}\right) \\
(6)\end{array}$ \\
\hline Chandra ACIS-S & 5636 & 2004 Dec 26 & $0.5-8$ & 7.63 & 4.96 \\
\hline XMM-Newton $\mathrm{PN} / \mathrm{MOS} 1+2$ & 0601420101 & 2009 Jul 25 & $0.5-10$ & $45.4 / 53.4$ & $14.6 / 3.76$ \\
\hline Swift-BAT & $\ldots$ & 2004-2010 & $14-100$ & 7340 & 0.00223 \\
\hline Swift-XRT & 00080731001 & 2014 May 24 & $0.5-10$ & 3.96 & 0.472 \\
\hline NuSTAR FPMA/FPMB & 60061362002 & 2014 May 24 & $3-50$ & $22.5 / 22.4$ & $2.44 / 1.94$ \\
\hline NuSTAR FPMA/FPMB & 60061362004 & 2014 Jun 30 & $3-50$ & $19.7 / 19.7$ & $2.32 / 2.36$ \\
\hline
\end{tabular}

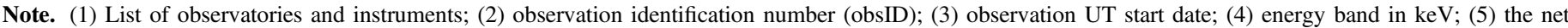
exposure time in ks; (6) net count rate for the AGN in the given energy band in units of $10^{-2} \mathrm{cts} \mathrm{s}^{-1}$.

data for NGC $5643 \mathrm{X}-1$, which is detected above $10 \mathrm{keV}$ for the first time.

The paper is organized as follows: we describe details of the $\mathrm{X}$-ray observations and data reduction of the AGN in Section 2, followed by the spectral fitting procedures and results in Section 3. In Section 4, we present the data analysis and results on NGC $5643 \mathrm{X}-1$. This is followed by a discussion in Section 5. The paper concludes with a summary in Section 6.

\section{OBSERVATIONS}

In this section, we describe the NuSTAR observations and data analysis procedures for the AGN. We also detail the archival Chandra, XMM-Newton, and Swift data that were used to facilitate our broadband spectral analysis. Details of these observations are provided in Table 1 , and the data reduction is described below.

\subsection{NUSTAR}

NuSTAR (Harrison et al. 2013), operating at $3-79 \mathrm{keV}$, is the first orbiting observatory with the ability to focus X-ray photons at $E>10 \mathrm{keV}$. It provides a two orders of magnitude improvement in sensitivity and over an order of magnitude improvement in angular resolution with respect to previous hard X-ray orbiting observatories. It consists of two co-aligned focal plane modules, FPMA and FPMB, each covering the same $12^{\prime} \times 12^{\prime}$ portion of the sky, and each comprised of four detectors placed in a $2 \times 2$ array. NUSTAR has an angular resolution of $18^{\prime \prime}$ FWHM with a half-power diameter of $\sim 1^{\prime}$, and energy resolutions (FWHM) of 0.4 and $0.9 \mathrm{keV}$ at 6 and $60 \mathrm{keV}$, respectively. These capabilities make NuSTAR an ideal instrument to characterize the spectral shape of heavily obscured AGNs in the local universe (e.g., Baloković et al. 2014; Bauer et al. 2014; Gandhi et al. 2014; Puccetti et al. 2014).

NGC 5643 was observed twice by NUSTAR in 2014-an initial observation with a nominal exposure time of $22.5 \mathrm{ks}$ taken in May, followed by an additional $19.7 \mathrm{ks}$ observation in June. The second observation was conducted to improve the S/ $\mathrm{N}$ of NGC 5643 as it fell near the detector gap in the first observation. The data were processed with the NuSTAR Data Analysis Software (NUSTARDAS) v1.4.1 within HEASOFT v6.15.1 with CALDB v20150316. Calibrated and cleaned event files were produced using the NUPIPELINE v0.4.3 script with standard filter flags. Spectra and response files were extracted using the NUPRODUCTS v0.2.5 task.
For each observation, the AGN spectra were extracted using a circular aperture region of $30^{\prime \prime}$-radius centered on its peak emission. The background photons were collected from polygon-shaped regions lying on the same detector as the source for the June observation, and from two adjacent detectors for the May observation (since the source fell close to the detector gap in this observation). We excluded background photons that lay within a circular region of $\sim 70^{\prime \prime}$-radius around the source to exclude emission from NGC $5643 \mathrm{X}-1$. Significant counts are detected at up to $50 \mathrm{keV}$ for the AGN. We show the combined FPMA+B images of the AGN and NGC $5643 \mathrm{X}-1$ in the 3-8, 8-24, and 24-50 keV bands in Figure 1.

\subsection{XMM-Newton}

NGC 5643 was also observed on two occasions by XMMNewton, for $10 \mathrm{ks}$ in 2003 and $55 \mathrm{ks}$ in 2009. In both cases, the observations were performed with the EPIC CCD cameras (PN, MOS1, MOS2), operated in full frame mode with the medium filter. The observations were discussed in detail in G04 and M13, respectively. For the AGN, we only extracted and used the spectra from the longer 2009 observation, as it has the highest $\mathrm{S} / \mathrm{N}$. Data were reduced within SAS v1.2, screened for flaring particle background as described in M13.

Source spectra were extracted within a $25^{\prime \prime}$-radius aperture centered on the AGN for all cameras. Background photons were selected from source-free circular regions of $100^{\prime \prime}$-radius on the same chip as the source. Patterns $0-4$ and $0-12$ were used for the PN and MOS spectra, respectively. The spectra from MOS1 and MOS2 were co-added, as the data were consistent with each other (see Section 3 for further details).

\subsection{Chandra}

NGC 5643 has only been observed once by Chandra. This observation was conducted in 2004 with the ACIS-S detector with an exposure time of $\sim 8 \mathrm{ks}$. The results of the observation were first published in Bianchi et al. (2006). We reprocessed the data to create event files with updated calibration modifications using the CIAO v4.6 pipeline following standard procedures.

Source counts were extracted using the SPECEXTRACT task in CIAO from a circular region of $25^{\prime \prime}$-radius centered on the AGN to match the XMM-Newton extraction region. The background was extracted from a source-free $30^{\prime \prime}$-radius circular region close to the source. 


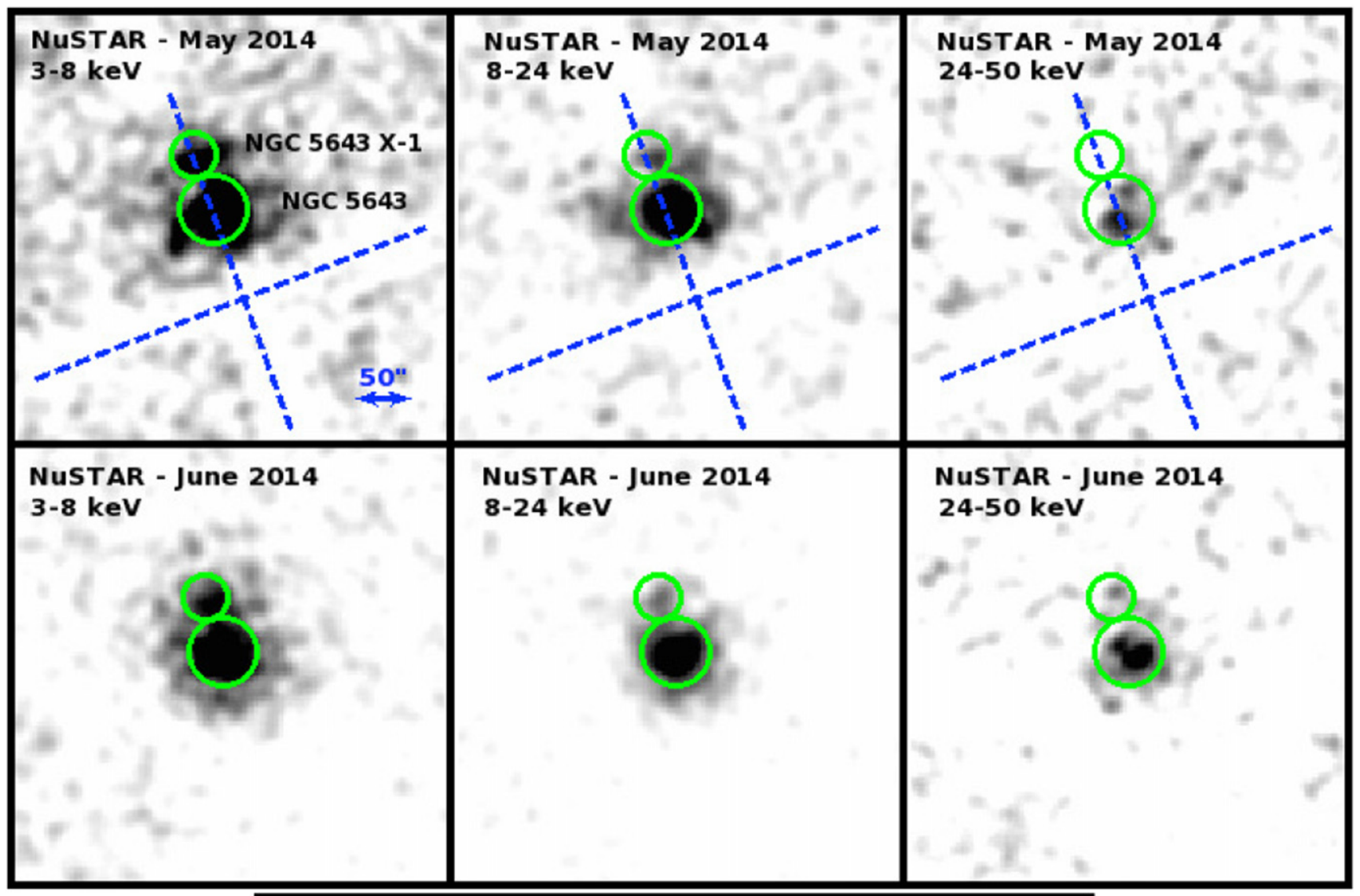

XMM-Newton Epic MOS 2 - July 2009

NGC 5643 X-1

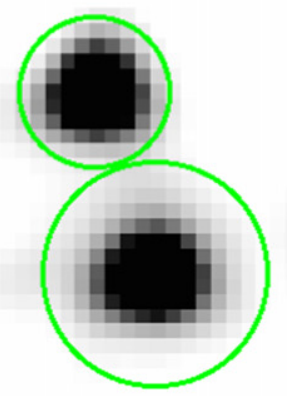

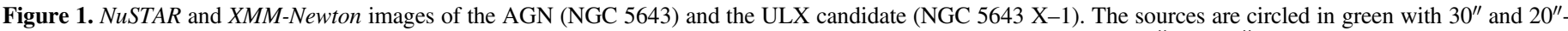

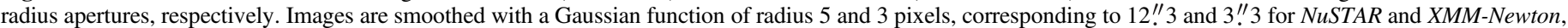

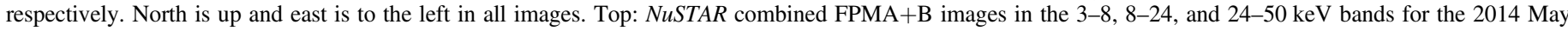

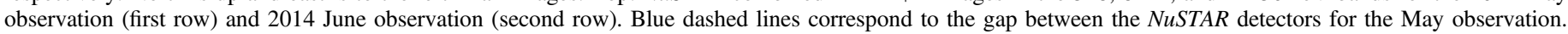
Bottom: XMM-Newton EPIC-MOS2 image (2009 July) in the 0.5-10 keV band, shown to provide a clearer view of the two sources at lower energies.

\subsection{Swift}

The first NuSTAR observation in 2014 May was accompanied by a $\sim 5 \mathrm{ks}$ Swift X-ray Telescope (XRT; Burrows et al. 2005) observation, which started approximately an hour after the NuSTAR observation began. The purpose of this observation was to provide simultaneous coverage for the soft
X-ray end of the spectrum $(\lesssim 3 \mathrm{keV})$ where the NUSTAR sensitivity drops off. The data were reduced using the XRTPIPELINE v0.13.0, which is part of the XRT Data Analysis Software (XRT-DAS) within HEASOFT. However, with only $\sim 20$ counts between 0.5 and $10 \mathrm{keV}$, the exposure is not long enough to provide additional constraints beyond those already obtained with NUSTAR, XMM-Newton, and Chandra. 
Table 2

X-Ray Spectral Fitting Results for the AGN in NGC 5643

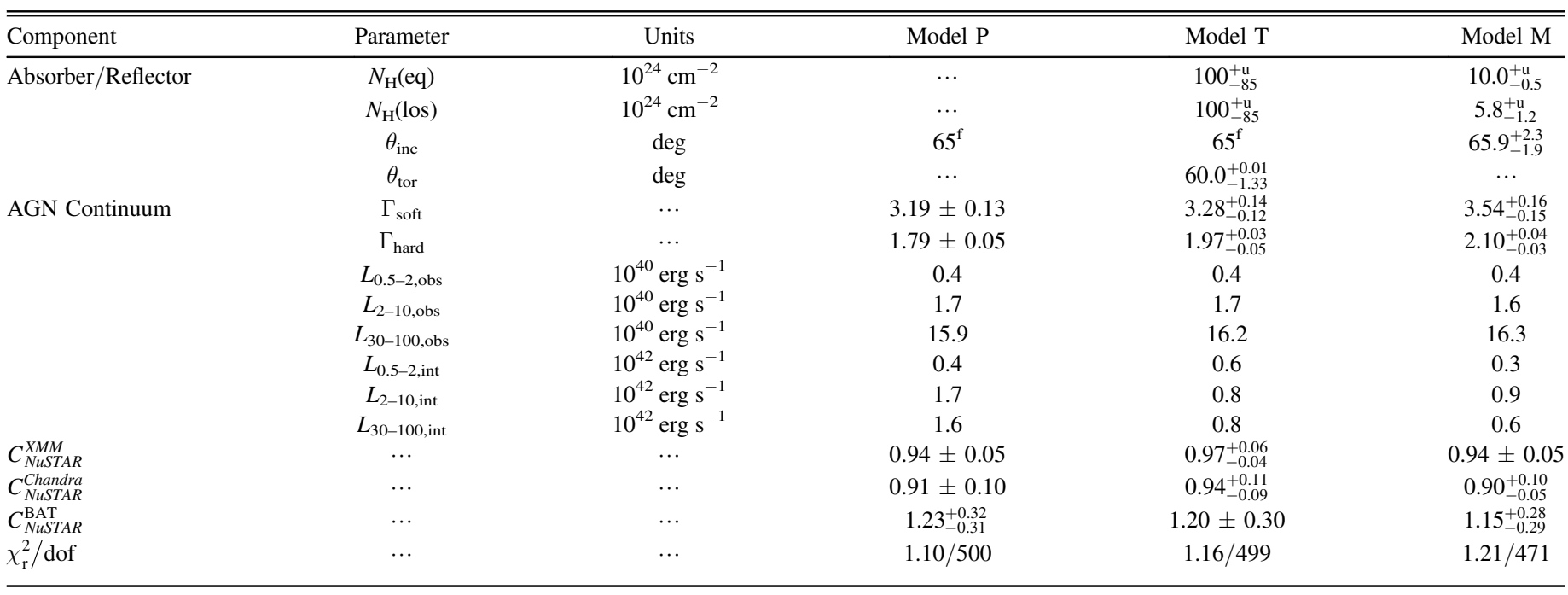

Notes. Best-fitting model parameters for Model P (PEXRAV model by Magdziarz \& Zdziarski 1995), Model T (ToRus model by Brightman \& Nandra 2011), and Model M (MYTonus model by Murphy \& Yaqoob 2009). Details of each model are described in Section 3.

${ }^{\mathrm{f}}$ Fixed.

${ }^{\mathrm{u}}$ Unconstrained.

Therefore, we only used these data to check for consistency with the XMM-Newton and Chandra data at $0.5-10 \mathrm{keV}$ (see Section 3).

The Burst Alert Telescope (BAT; Barthelmy et al. 2005) on board Swift has been continuously monitoring the sky at 14-195 keV, producing images of a large number of hard X-ray sources thanks to its wide field of view and large sky coverage. We used the stacked 70-month spectrum and its associated response file downloaded from the Swift-BAT 70-month Hard X-ray Survey Source Catalog (Baumgartner et al. 2013) to provide X-ray constraints above the NuSTAR band. ${ }^{27}$ NGC 5643 is detected at the $5.4 \sigma$ significance level with significant counts up to $\sim 100 \mathrm{keV}$.

\section{BROADBAND SPECTRAL MODELING OF THE AGN}

We describe the broadband X-ray spectral analysis of the AGN in this section. The analysis was carried out using XSPEC v12.8.2. ${ }^{28}$ All spectra were binned to a minimum of 25 counts per bin to allow the use of $\chi^{2}$ statistics. Absorption through a fixed Galactic column along the line of sight, $N_{\mathrm{H}}^{\mathrm{Gal}}=8.01 \times 10^{20} \mathrm{~cm}^{-2}$ (Kalberla et al. 2005), was included in all spectral fits using the XSPEC model "PHABS," and solar abundances were assumed for all models. All errors are quoted at $90 \%$ confidence, unless stated otherwise. Details of the main results are summarized in Table 2, and the best-fit spectra are shown in Figure 2.

We began our spectral modeling with an examination of the NUSTAR data alone. We started by modeling the spectra of each of the NuSTAR observations in the $3-50 \mathrm{keV}$ band using a simple absorbed power-law model with the column density fixed to the Galactic value. A prominent excess of counts just above $6 \mathrm{keV}$ was observed in both spectra, suggesting the

\footnotetext{
27 The Swift-BAT 70-month Hard X-ray Survey Source Catalog is available online at http://swift.gsfc.nasa.gov/results/bs70mon/

${ }^{28}$ The XSPEC manual can be downloaded from http://heasarc.gsfc.nasa.gov/ xanadu/xspec/XspecManual.pdf
}

presence of $\mathrm{Fe} \mathrm{K} \alpha$ emission. The parameters returned by the two spectra were consistent with each other, indicating that there are no significant differences between the two observations. Therefore, we co-added the spectra for each FPM using the ADDASCASPEC script (the same test and procedures were also done for the two XMM-Newton MOS spectra).

We then modeled the combined NuSTAR spectra in the 3-50 keV band with an absorbed power-law model (column density fixed to $N_{\mathrm{H}}^{\mathrm{Gal}}$ ) and a Gaussian line at $E \approx 6.4 \mathrm{keV}$. The model measured a photon index and $\mathrm{Fe} \mathrm{K} \alpha \mathrm{EW}$ of $\Gamma=0.55 \pm$ 0.07 and $\mathrm{EW}_{\mathrm{Fe}}=2.22_{-0.34}^{+0.35} \mathrm{keV}$, respectively, with a fit statistic of $\chi^{2}=115$ for 76 degrees of freedom (dof). The line energy is centered at $E=6.36 \pm 0.04$ (statistical) \pm 0.04 (systematic; Madsen et al. 2015) keV, consistent with neutral Fe $\mathrm{K} \alpha$ emission. The very flat $\Gamma$ and large $\mathrm{EW}_{\mathrm{Fe}}$, which were also found in previous observations, are characteristic signatures of CT absorption and reflection from optically thick cold gas.

We checked for variability in the flux of the AGN in the 0.5-10 keV band by comparing the XMM-Newton and Chandra data with the Swift-XRT data. The fluxes of all spectra are consistent with each other, $f_{0.5-10} \sim 10^{-12} \mathrm{erg} \mathrm{s}^{-1} \mathrm{~cm}^{-2}$, suggesting that there are no significant differences between the three observations. We also checked for variability at hard $\mathrm{X}$-ray energies by constructing power spectra from the 3-50 keV NuSTAR light curves from the two observations. These were then compared to the power spectra expected for pure Poisson noise and for the expected transmitted component variability observed in an unobscured AGN with a similar black hole mass and accretion rate (see Arévalo et al. 2014 for further details). No evidence for variability was found, however, given the low count rates, this test is not very sensitive. Given that the NUSTAR spectra from the two observations are consistent with each other, we assumed that the AGN has not varied.

We therefore proceeded to fit the XMM-Newton and Chandra spectra combined with the NuSTAR and SwiftBAT spectra using more detailed physical models to better 

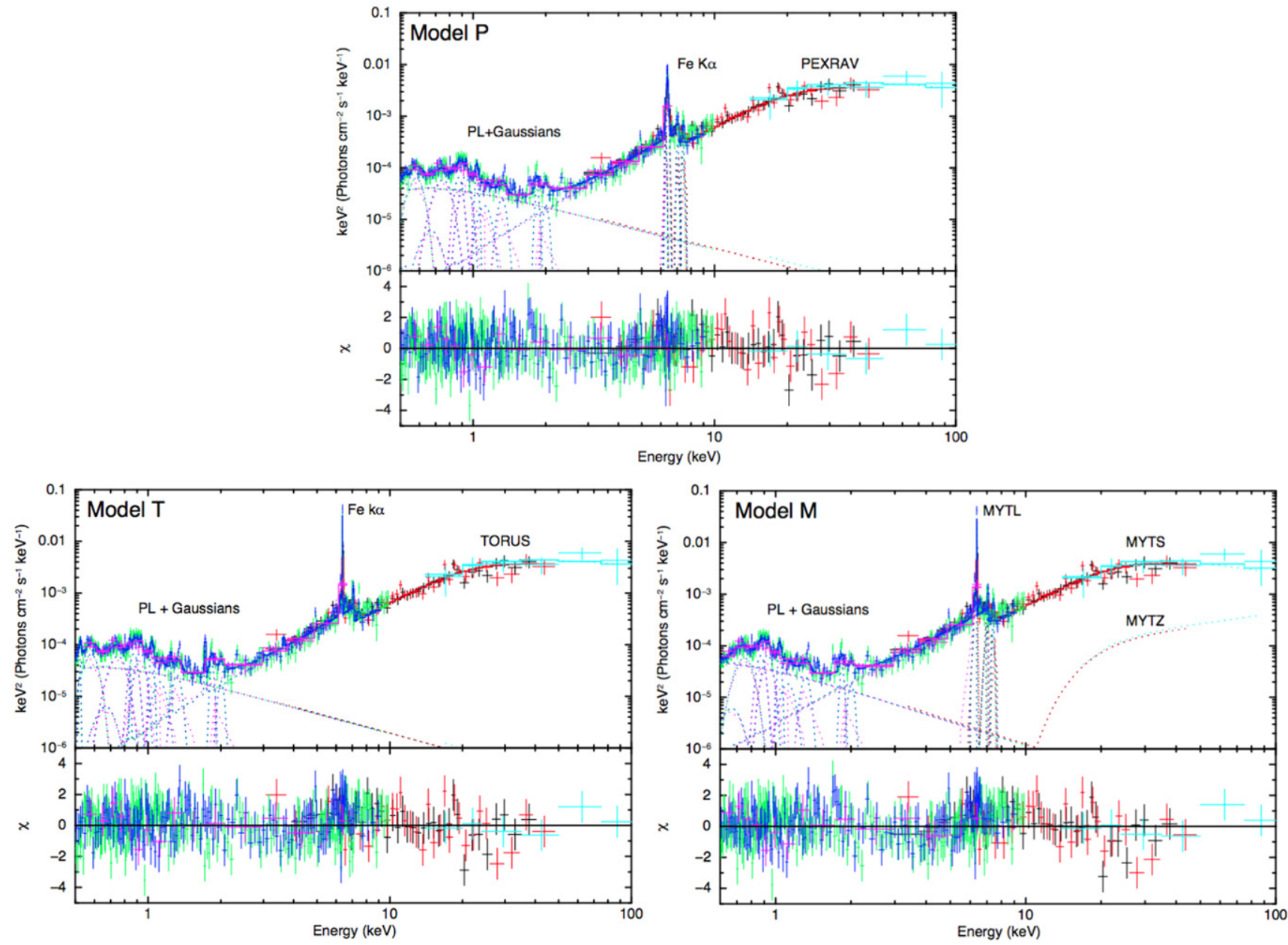

Figure 2. Best-fit models to the combined NuSTAR, Chandra, XMM-Newton, and Swift-BAT data of the AGN—Model P (top), Model T (bottom left), and Model M (bottom right). Model P and Model T were fitted between 0.5 and $100 \mathrm{keV}$, and Model $\mathrm{M}$ was fitted between 0.6 and $100 \mathrm{keV}$ since we found strong residuals near $\sim 0.5 \mathrm{keV}$ for this model. The lower energy data $(\lesssim 2 \mathrm{keV})$ were modeled using a soft power-law (PL) and Gaussian lines to simulate photoionization by the AGN. The top panels of each plot show the unfolded model in $E F_{E}$ units, while the bottom panels show the fit residuals in terms of sigma with error bars of size one. Color scheme: black (NUSTAR FPMA), red (NuSTAR FPMB), blue (XMM-Newton PN), green (XMM-Newton MOS), purple (Chandra), and cyan (Swift-BAT).

characterize the broadband spectrum of the AGN. We describe the details and results of each model used: Model P (PEXRAV model by Magdziarz \& Zdziarski 1995), Model T (TORus model by Brightman \& Nandra 2011), and Model M (MYToRus model by Murphy \& Yaqoob 2009), in Sections 3.1-3.3, respectively. In addition to these models, we added extra components required to provide a good fit to the data, briefly described below.

The soft energy $(\lesssim 2 \mathrm{keV})$ part of the spectra covered by $X M M$-Newton and Chandra is dominated by emission from photoionized material (M13). We modeled this emission based on M13 using a soft power-law ${ }^{29}$ and 10 Gaussian components (9 for Model M; see Section 3.3) to model the emission lines (refer to Table 1 in M13). We also added four and one more Gaussian component(s) to Model P and Model M, respectively, to model the fluorescence lines emitted at $E \gtrsim 2 \mathrm{keV}$ that are not included in these models (see Sections 3.1 and 3.3 respectively).

Cross-calibration uncertainties between each observatory with respect to NUSTAR were included as free parameters

\footnotetext{
29 The soft power-law used to model the unresolved $\leq 2 \mathrm{keV}$ emission also includes the scattered power-law component, which is commonly used to simulate the AGN emission scattered into our line of sight by diffuse hot gas.
}

(CONSTANT, C). Initially, we left both the XMM-Newton EPICMOS, and PN cameras constants free to vary. However, we found that their values are consistent with each other and decided to tie them together in the final fittings of all models.

Our three models can be described as follows:

$$
\begin{aligned}
\text { Model } \mathrm{P}= & \text { CONSTANT } \times \text { PHABS } \times(\text { POW } \\
& + \text { PEXRAV }+14 \times \text { ZGAUSS }), \\
\text { Model } \mathrm{T}= & \text { CONSTANT } \times \text { PHABS } \times(\text { POW } \\
& + \text { TORUS }+10 \times \text { ZGAUSS }) . \\
\text { Model } \mathrm{M}= & \text { CONSTANT } \times \text { PHABS } \times(\text { POW } \\
+ & \text { ZPOW } \times \text { MYTZ }+ \text { MYTS }+ \text { MYTL } \\
+ & 10 \times \text { ZGAUSS }),
\end{aligned}
$$

\subsection{Model P}

In our fitting of the AGN broadband spectrum, we first consider the PEXRAV reflection model ("Model P"; Magdziarz \& Zdziarski 1995). This model has commonly been used to model reflection-dominated spectra. However, it assumes reflection off a slab geometry with an infinite column density, which is 
unlikely to represent the true geometry of the AGN torus. It also does not incorporate fluorescence emission lines expected from a CTAGN, such as the $\mathrm{Fe} \mathrm{K} \alpha$ and $\mathrm{K} \beta$ lines. Because of these limitations, we also fitted the spectra of the AGN using more physically motivated reflection/obscuration models as described in Sections 3.2 and 3.3 However, Model P can provide useful comparison with the other reflection models and previous studies of this source, as well as other CT sources.

We started by fixing all the line energies and normalizations of the soft Gaussian components to the values presented in M13. We also fixed the redshift at $z=0.0040$. Initially, we set the intrinsic line widths to $\sigma=50 \mathrm{eV}$, as we expect them to be unresolved. We then allowed the widths for each line to vary in turn, to improve the fit. For those that returned values deviating significantly from $50 \mathrm{eV}$, we fixed them to this new value.

We added several more Gaussian components to account for the $\mathrm{Fe} \mathrm{K} \alpha$ and $\mathrm{Fe} \mathrm{K} \beta$ lines, the $\mathrm{Ni} \mathrm{K} \alpha$ line, and also the Compton shoulder associated with the $\mathrm{Fe} \mathrm{K} \alpha$ line, which are not included in the model. ${ }^{30}$ The parameters were also fixed to values obtained in M13, except for the line energy and normalization of the $\mathrm{Fe} \mathrm{K} \alpha$ line, which were allowed to vary throughout. We fixed the inclination of the reflector to $\theta_{\text {inc }}=65^{\circ}$, which is equal to that modeled by Fischer et al. (2013) for NGC 5643 (with an uncertainty of $\pm 5^{\circ}$ ), based on mapping the kinematics of the $\mathrm{H} \alpha$ and [O III] narrow line region (NLR) emission observed by the Hubble Space Telescope. The reflection scaling factor was fixed to $R=-1$ to simulate a reflection-dominated spectrum. Model $\mathrm{P}$ provides a good fit to the data $\left(\chi^{2} /\right.$ dof $\left.=551 / 500\right)$, indicating that the spectra can be well-fitted by a pure reflection model without the need for any direct component.

The cross-calibration constants of XMM-Newton, Chandra and Swift-BAT relative to $N U S T A R$ are consistent with each other with $C \sim 1$ within the statistical errors (see Table 2), indicating that the spectra are in good agreement with each other (see also Madsen et al. 2015 for the current crosscalibration status between different X-ray observatories and instruments with respect to $N u S T A R)$. We estimated the intrinsic X-ray luminosities from this model by assuming that the observed luminosities at $0.5-10 \mathrm{keV}$ and $30-100 \mathrm{keV}$ are about $1 \%$ and $10 \%$ of the intrinsic luminosities at these energy bands, respectively (see Table 2). These inferred fractions were taken from detailed studies of NGC 1068 and have been widely used to estimate the intrinsic luminosities of other reflectiondominated AGN (e.g., Matt et al. 1997; Baloković et al. 2014).

\subsection{Model T}

We then proceeded to fit the AGN broadband spectrum using more physically motivated models, starting with the TORUS model by Brightman \& Nandra (2011) (Model T). This model simulates obscuration by a medium with a conical section cut from a sphere, with a variable biconical polar opening angle $\left(\theta_{\text {tor }}\right)$ ranging between $26^{\circ}$ and $84^{\circ}$. Small values of $\theta_{\text {tor }}$ correspond to a geometrically thick torus, while large values indicate a geometrically thin torus. The line of sight $N_{\mathrm{H}}(\mathrm{los})$ through the torus, which is equal to the equatorial column density $N_{\mathrm{H}}(\mathrm{eq})$, extends up to $10^{26} \mathrm{~cm}^{-2}$, allowing investigations of the most extreme obscuration, and is defined such that

\footnotetext{
${ }^{30}$ We note that modeling the Compton shoulder using a Gaussian component is incorrect since it is not exactly Gaussian, but we adopted this approach as an approximate parameterization for this particular model.
}

it is independent of the inclination angle $\left(\theta_{\text {inc }}\right)$. The model is defined between 0.1 and $320 \mathrm{keV}$, and self-consistently predicts lines commonly found in obscured AGNs, such as $\mathrm{Fe} \mathrm{K} \alpha, \mathrm{Fe}$ $\mathrm{K} \beta$ and also $\mathrm{K} \alpha$ emission from several other elements $(\mathrm{C}, \mathrm{O}$, $\mathrm{Ne}, \mathrm{Mg}, \mathrm{Si}, \mathrm{Ar}, \mathrm{Ca}, \mathrm{Cr}, \mathrm{Fe}$, and $\mathrm{Ni}$ ).

The soft components of the spectrum $(\lesssim 2 \mathrm{keV})$ were modeled as described earlier in Sections 3 and 3.1. Since $\theta_{\text {inc }}$ and $\theta_{\text {tor }}$ could not be constrained simultaneously, we fixed $\theta_{\text {inc }}$ to $65^{\circ}$ (Fischer et al. 2013). This model also yields a good fit to the data $\left(\chi^{2} /\right.$ dof $\left.=579 / 499\right)$, with most fit residuals lying at the soft energies $(\lesssim 2 \mathrm{keV})$ and around the iron line emission ( $\sim 6-7.5 \mathrm{keV}$; e.g., $\chi^{2} /$ dof $=447 / 417$ when fitting the model by ignoring the data around the iron line emission). The fitted intrinsic continuum power-law emission from this model has a photon index of $\Gamma=1.97_{-0.05}^{+0.03}$ and is absorbed by a column density of $N_{\mathrm{H}}(\mathrm{los})>1.5 \times 10^{25} \mathrm{~cm}^{-2}$; i.e., heavily Comptonthick. The upper limit is unconstrained, with values of up to $10^{26} \mathrm{~cm}^{-2}$ allowed by the model. We found that the best-fit $\theta_{\text {tor }}$ measured by this model is $\approx 60^{\circ}$. As with Model P, the crosscalibration constants of each observation with respect to NUSTAR are consistent with 1 . We inferred the intrinsic luminosities from this model based upon the best-fit parameters obtained, as presented in Table 2.

We also tried to fit the spectrum using different $\theta_{\text {inc }}$ to see how the key parameters would change. Setting $\theta_{\text {inc }}$ to a lower value of $45^{\circ}$ produced an unacceptable fit $\left(\chi^{2} /\right.$ dof $\left.=3989 / 499\right)$. Fixing $\theta_{\text {inc }}$ to a higher value of $85^{\circ}$ to simulate a near edge-on torus inclination, however, provides a slight improvement to the fit $\left(\chi^{2} /\right.$ dof $\left.=549 / 499\right)$. The column density obtained is consistent with what was inferred before, but is more constrained, $N_{\mathrm{H}}(\mathrm{los})=1.8_{-0.6}^{+4.3} \times 10^{25} \mathrm{~cm}^{-2}$. The photon index is slightly lower, $\Gamma=1.75_{-0.03}^{+0.01}$, and the opening angle of the torus suggests a surprisingly thin torus, $\theta_{\text {tor }}=78.5_{-0.74}^{+0.01}$ degrees. However, we note that Liu \& Li (2015) claim that for an edge-on torus inclination, this model overestimates the reflection component, and therefore the parameters obtained may not be reliable. We thus favor the best-fitting model solution using $\theta_{\text {inc }}=65^{\circ}$.

A closer look at the fitted spectrum between 6 and $7.5 \mathrm{keV}$ (Figure 3) indicates that the model overpredicts the data at $\approx 6.3 \mathrm{keV}$, which encompass the Compton shoulder associated with the $\mathrm{Fe} \mathrm{K} \alpha$ line emission, and underpredicts the data at $\approx 6.4-6.8 \mathrm{keV}$, which include the $\mathrm{Fe} \mathrm{K} \alpha$ line emission. We investigated whether the observed residuals could be caused by the presence of emission lines at 6.70 and $6.96 \mathrm{keV}$ corresponding to ionized iron by adding Gaussian components at these energies. However, we found that the normalizations of the components are consistent with zero. We also tried to add a line smoothing component to the model (GSMOоTH; energy index, $\alpha=1$ ) to see if the statistics could be further improved, but the fit indicated that this is not required $\left(\sigma_{\text {gsmooth }} \lesssim 40 \mathrm{eV}\right)$. We noticed that the residuals are dominated by the $X M M$ Newton data and therefore checked whether or not these could be caused by a shift in the energy scale of the data. Indeed we found that, applying an $\sim+11 \mathrm{eV}$ offset in the PN data can improve the overall quality of the fit $\left(\chi^{2} /\right.$ dof $\left.=551 / 497\right)$ and diminish the residuals around the iron line complex (see also Bauer et al. 2014 for similar finding). However, this energy shift is consistent with the current calibration uncertainty of $X M M-N$ wton $^{31}$, and the final results of the fitting are

\footnotetext{
31 The current calibration documentation of $X M M-N e w t o n$ EPIC cameras can be downloaded from http://xmm2.esac.esa.int/docs/documents/CAL-TN0018.pdf.
} 


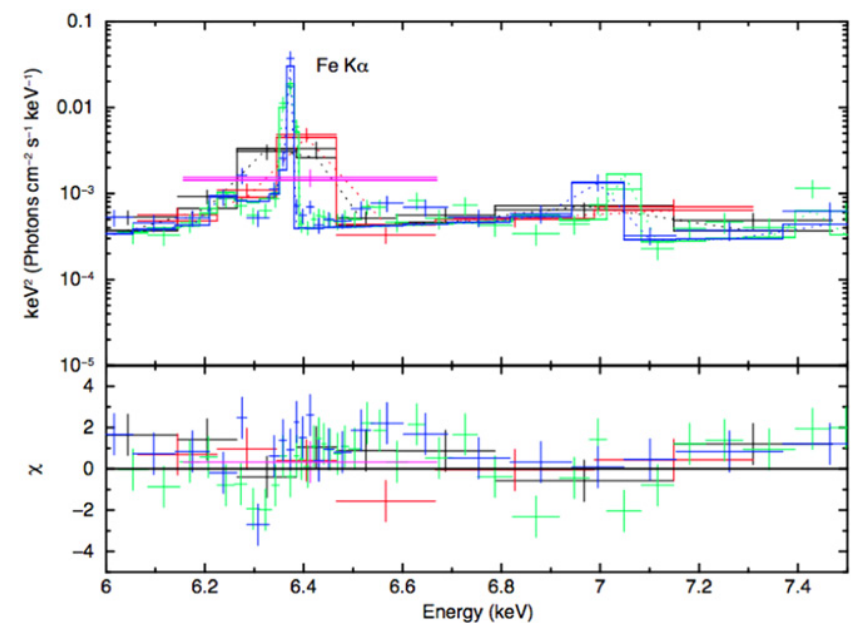

Figure 3. Zoom-in of the spectral fit for Model T between 6 and $7.5 \mathrm{keV}$ showing the asymmetric residuals around the iron line emission; the $x$-axis has been de-redshifted to show the rest-frame energy. The color scheme of the data and the model are the same as Figure 2. The residuals are dominated by the $X M M-N e w t o n$ data at $\approx 6.3-6.8 \mathrm{keV}$, with the model overestimating the emission at $\approx 6.3 \mathrm{keV}$, and underpredicting the data at $\approx 6.4-6.8 \mathrm{keV}$. These residuals are diminished when applying an $\sim+11 \mathrm{eV}$ offset in the $X M M$ Newton PN data.

consistent with those presented in Table 2. Therefore, we decided not to apply this energy shift to the XMM-Newton PN data in the final fitting of all models.

\subsection{Model $M$}

We next fitted the AGN spectrum using the MYTorus model by Murphy \& Yaqoob (2009; Model M). This model simulates obscuration toward an AGN using a toroidal absorber geometry (circular cross-section) with a fixed opening angle $\theta_{\text {tor }}=60^{\circ}$. The advantage of this model over Model $\mathrm{T}$ is that it allows the separation of the direct (MYTZ), scattered (MYTS), and line emission (MYTL) components, allowing more freedom to explore complex geometries in the modeling. On the other hand, the line of sight $N_{\mathrm{H}}(\mathrm{los})$ is tied to the inclination angle and the equatorial $N_{\mathrm{H}}(\mathrm{eq})$, which is available only up to an absorbing column density of $N_{\mathrm{H}}(\mathrm{eq})=10^{25} \mathrm{~cm}^{-2}$. The MYTL component self-consistently includes neutral $\mathrm{Fe} \mathrm{K} \alpha$ and $\mathrm{Fe} \mathrm{K} \beta$ fluorescence lines, and the associated Compton shoulders. The model is defined between 0.5 and $500 \mathrm{keV}$, but we noticed strong residuals in our fit near the lower energy threshold, and therefore restricted our fits to above $0.6 \mathrm{keV}$ for this model. ${ }^{32}$

We fitted the AGN spectrum using the simplest version of the MYToRus model, which couples all of the parameters of the scattered and fluorescence line components to the direct continuum component. We added an extra Gaussian component to account for the $\mathrm{Ni} \mathrm{K} \alpha$ line that is not included in the model (parameters were fixed to values obtained in M13). The relative normalizations of MYTS $\left(A_{S}\right)$ and MYTL $\left(A_{L}\right)$ with respect to MYTZ $\left(A_{Z}\right)$ were set to 1 . Model M also fits the data well with $\chi^{2} /$ dof $=569 / 471$. The best-fitting global column density inferred is $N_{\mathrm{H}}(\mathrm{eq})=10.0_{-0.5}^{+\mathrm{u}} \times 10^{24} \mathrm{~cm}^{-2}$, which is at the upper limit of the MYToRus model. Reassuringly, the bestfitting inclination angle is $\theta_{\text {inc }}=65.9_{-1.9}^{+2.3}$ degrees, consistent with that determined by Fischer et al. (2013). However, we

\footnotetext{
${ }^{32}$ Since the fit for Model $\mathrm{M}$ was restricted to $E \geqslant 0.6 \mathrm{keV}$, a Gaussian component that was added to model the emission line at $E \approx 0.58 \mathrm{keV}$ (M13) in Model P and T was excluded for this model.
}

note that the MYToRus model does not allow for complete freedom in the fitting of the inclination angles. Due to the assumption made for $\theta_{\text {tor }}$, the model treats a torus with $\theta_{\text {inc }}<$ $60^{\circ}$ as unobscured, and as a result, the best-fit $\theta_{\text {inc }}$ measured by the model for obscured AGNs are usually in the range of $\approx 60^{\circ}-70^{\circ}$. The $N_{\mathrm{H}}(\mathrm{los})$ measured from this model is well within the CT regime, with an unconstrained upper limit; i.e., $N_{\mathrm{H}}(\mathrm{los})=5.8_{-1.2}^{+\mathrm{u}} \times 10^{24} \mathrm{~cm}^{-2}$. Similar to Model $\mathrm{T}$, the residuals of the fit are dominated by the soft energy emission and the emission around the iron line, which can be improved if an energy offset is allowed in the XMM-Newton PN data (see Section 3.2). All parameters obtained by Model M, including the cross-calibration normalization constants and intrinsic luminosities of the AGN, agree very well with Model T (see Table 2).

\subsection{The SPHERE Model}

In addition to the TORUS model described in Section 3.2, Brightman \& Nandra (2011) also present a model in which the source is fully covered by a spherical geometry $\left(\theta_{\text {tor }}=0^{\circ}\right)$ with variable elemental and iron abundances with respect to hydrogen. This model is referred to as the SPHERE model. We tried to fit the spectra using this model. However, the fit was very poor $\left(\chi^{2} /\right.$ dof $\left.=1169 / 501\right)$ and difficult to constrain. Leaving the metal abundances free to vary, which is allowed in this model, did improve the fit slightly, but the fit was still poor $\left(\chi^{2} /\right.$ dof $\left.=848 / 499\right)$, and returned very low values of $N_{\mathrm{H}}$ and $\Gamma$, and a high $\mathrm{Fe}$ abundance $(\sim 7)$. Therefore, we will not discuss this model any further.

\section{NGC $5643 \mathrm{X}-1$}

In addition to the AGN, we also analyzed the NUSTAR data for the ULX candidate, NGC 5643 X-1. ULXs are off-nuclear point sources with X-ray luminosities exceeding the Eddington limit for the typical $\sim 10 M_{\odot}$ stellar-remnant black holes observed in Galactic black hole binaries (e.g., Orosz 2003); i.e., $L_{\mathrm{X}} \gtrsim 10^{39} \mathrm{erg} \mathrm{s}^{-1}$, potentially implying exotic superEddington accretion (e.g., Poutanen et al. 2007). While the majority of ULXs have luminosities of the order of $10^{39} \mathrm{erg} \mathrm{s}^{-1}$ (Swartz et al. 2011; Walton et al. 2011b), a small subset of the population has been observed to have $L_{\mathrm{X}}>10^{40} \mathrm{erg} \mathrm{s}^{-1}$ (Walton et al. 2011a; Jonker et al. 2012; Sutton et al. 2012). Given their luminosities, it has been suggested that these more luminous sources might be good candidates for hosting intermediate mass black holes $\left(10^{2} \lesssim M_{\odot} \lesssim 10^{5}\right.$; e.g., Miller et al. 2004; Strohmayer 2009) accreting at sub-Eddington rates. With a luminosity of $L_{0.5-10}=2.6 \times 10^{40} \mathrm{erg} \mathrm{s}^{-1}$ as measured by G04, NGC $5643 \mathrm{X}-1$ would therefore be a member of this latter population.

We extracted the NuSTAR spectra of NGC $5643 \mathrm{X}-1$ by defining a smaller circular region of $20^{\prime \prime}$-radius around the source using the AGN as a reference point for its relative position. For the background region, we used an annular segment centered on the AGN (excluding a region of $\sim 50^{\prime \prime}$ radius around NGC $5643 \mathrm{X}-1$ which accounts for the $\sim 70 \%$ encircled energy fraction), and with the same radial distance (relative to the AGN position) and width as the source region. This background region was designed to account for contamination by the AGN. We then determined the significance of the NUSTAR detection by calculating a no-source probability assuming binomial statistics $(P>1 \%$ for non- 
detection; i.e., $\lesssim 2.6 \sigma$, following other $N u S T A R$ studies of faint sources-e.g., Luo et al. 2013; Lansbury et al. 2014; Stern et al. 2014). While the source is significantly detected in both of the NuSTAR observations in the $3-8 \mathrm{keV}$ band, it is only significantly detected in the second observation in the $8-24 \mathrm{keV}$ band $\left(P=2.21 \times 10^{-6}\right.$ in the combined FPMA $+\mathrm{B}$ image).

We next combined the data from the two FPMs for each epoch and binned the spectra to a minimum of 5 counts per bin due to low counts. We then fitted the spectra from each epoch using a simple power-law model, absorbed by the Galactic column. The spectral fitting parameters were calculated using C-statistics, appropriate for low count statistics (Cash 1979). Although the source is not formally detected in the $8-24 \mathrm{keV}$ band in the first epoch, we modeled the spectrum including the data point in this band to provide a better constraint to the fit. We found that the spectra of the source during the first and second epoch are consistent with each other, $\Gamma=2.7_{-0.5}^{+0.6}$ and $\Gamma=1.7_{-0.5}^{+0.6}, f_{3-24}=3.6_{-0.8}^{+0.9} \times 10^{-13} \mathrm{erg} \mathrm{s}^{-1} \mathrm{~cm}^{-2}$, and $3.8_{-1.0}^{+1.4} \times 10^{-13} \mathrm{erg} \mathrm{s}^{-1} \mathrm{~cm}^{-2}$, respectively, suggesting that the source has not varied significantly between the two observations. The lack of a detection at $8-24 \mathrm{keV}$ in the first epoch may be due to the location of NGC $5643 \mathrm{X}-1$ relative to the NuSTAR detector gap (Figure 1), which reduced the overall sensitivity of the data.

Motivated by the lack of significant flux variability, we therefore combined the NUSTAR spectra of NGC $5643 \mathrm{X}-1$ from the two epochs and fitted the total spectrum with the Swift-XRT data that were taken simultaneously with the first epoch. We reduced the Swift-XRT data as detailed in Section 2.4, and extracted the spectrum using a $20^{\prime \prime}$-radius circular source region to match the NuSTAR extraction region. The background photons were selected from a source-free $100^{\prime \prime}$-radius circular region close to the source. We grouped the data to a minimum of 40 and 20 counts per bin for NuSTAR and Swift-XRT, respectively, allowing the use of $\chi^{2}$ statistics.

We first fitted the broadband spectrum of NGC $5643 \mathrm{X}-1$ over $0.5-24 \mathrm{keV}$ using a simple power-law model absorbed by a column density intrinsic to the host galaxy (TBABS) in addition to the Galactic absorption. We fixed the cross-normalization constants between NuSTAR and Swift-XRT to 1. The fit is acceptable with $\chi^{2} /$ dof $=9.21 / 7$. The spectrum seems to drop off at $E \approx 11 \mathrm{keV}$, which could be an indication of a spectral cutoff at around $10 \mathrm{keV}$, as found for other NuSTAR-observed ULXs (e.g., Bachetti et al. 2013; Walton et al. 2013, 2014; Rana et al. 2015). If shown to be statistically significant, this would be the first time that such a cutoff is observed for NGC $5643 \mathrm{X}-1$. We therefore proceeded to fit the spectrum using an absorbed cutoff power-law model. The fit is also acceptable $\left(\chi^{2} /\right.$ dof $\left.=8.46 / 6\right)$, and returned a cutoff energy of $E_{\text {cut }}=10.7_{-7.6}^{+\mathrm{u}} \mathrm{keV}$. We also fitted the spectra using a multicolor blackbody accretion disk model with a variable temperature disk profile, DISKPBB (Mineshige et al. 1994), which is commonly used to model ULX spectra. This model provides a marginal improvement to the fit over the simple and cutoff power-law model $\left(\chi^{2} /\right.$ dof $\left.=7.67 / 6\right)$. The measured temperature profile, $p$, is less than that expected for a thin disk $(p<0.75$; Shakura \& Sunyaev 1973), consistent with an accretion disk in which advection of radiation is important, as expected at very high accretion rates where radiation pressure dominates and modifies the structure of the disk (e.g., Abramowicz et al. 1988). This would be consistent with
NGC $5643 \mathrm{X}-1$ exhibiting a high-Eddington rate accretion onto a stellar-remnant black hole. The luminosity inferred by this model is $L_{0.5-24}=1.01_{-0.20}^{+0.36} \times 10^{40} \mathrm{erg} \mathrm{s}^{-1}$, assuming that NGC $5643 \mathrm{X}-1$ is at the distance of NGC 5643.

To obtain a better constraint to the broadband spectral fit of NGC 5643 X-1, we fitted the Swift-XRT and NuSTAR spectra simultaneously with the 2009 XMM-Newton spectrum of the source, in which M13 measured a luminosity of $L_{2-10}=(8.11 \pm 0.23) \times 10^{39} \mathrm{erg} \mathrm{s}^{-1}$, consistent with that inferred by our Swift-XRT $+N u S T A R$ best-fit DISKPBB model $L_{2-10}=7.53_{-1.39}^{+1.21} \times 10^{39} \mathrm{erg} \mathrm{s}^{-1}$. Data were reduced as described in Section 2.2. Because NGC $5643 \mathrm{X}-1$ fell on the detector gap in the PN camera, we only extracted the spectra of the source from the two MOS cameras, and co-added the spectra together. We extracted the spectra using a $20^{\prime \prime}$-radius circular source region, and a 45"-radius circular background region. We left the cross-normalization between the XMMNewton spectrum relative to NUSTAR, $C_{N u S T A R}^{X M M}$, free to vary.

We found that both the cutoff power-law and DISKPBB models provide better fits to the $S$ wift-XRT $+X M M+N u S T A R$ data than a simple power-law model $\left(\chi^{2} /\right.$ dof $=93.6 / 91,90.2 / 91$, and $99.0 / 92$, respectively). The F-test null hypothesis probability between the simple power-law and cutoff power-law model is 0.023 . This low value is strongly suggestive of the need for the high energy cutoff, though deeper observation will be required to validate this. $C_{N u S T A R}^{X M M}$ measured by the two models are, within the uncertainties, consistent with 1 , providing further evidence that the spectra are in good agreement with each other. The cutoff power-law model measured a cutoff energy of $E_{\text {cut }}=16.3_{-8.9}^{+35.9} \mathrm{keV}$, and the DISKPBB model inferred a temperature disk profile, $p=0.55 \pm 0.01$, and an inner disk temperature, $T_{\text {in }}=4.98_{-1.80}^{+2.46} \mathrm{keV}$. This temperature is consistent with that measured for some ULXs when fitting their whole broadband spectrum with just a single disk component (e.g., Walton et al. 2014; Rana et al. 2015). The best-fitting parameters for all models are presented in Table 3 , and the broadband Swift-XRT $+X M M+N u S T A R$ spectrum of NGC $5643 \mathrm{X}-1$ fitted by the best-fit model, namely DISKPBB, is shown in Figure 4.

Strong X-ray luminosity variations are often observed in ULXs. Motivated by this, we also reduced the 2003 XMMNewton data (ObsID 0140950101; UT 2003 February 08), as well as the Chandra data, and fitted the $3-8 \mathrm{keV}$ spectra of NGC 5643 X-1 using a simple power-law model absorbed by the Galactic column, to infer its $3-8 \mathrm{keV}$ luminosities at these epochs. In addition to these observations, the galaxy has also been observed by Swift-XRT between 2008 and 2013 on $\sim 30$ occasions (mostly $\sim 1 \mathrm{ks}$ exposure time), with most observations close to each other in 2013 targeted on an ongoing supernova in the galaxy. For simplicity, for each year in which there are multiple Swift-XRT observations of the source, we only analyzed the data with the highest nominal exposure time to provide the best estimate of NGC $5643 \mathrm{X}-1$ luminosity in that particular year (ObsID 00037275001, 00037275002, 00037275004, and 00032724009; UT 2008 March 16, 2009 September 20, 2010 October 01 and 2013 February 26, respectively). Among these observations, NGC $5643 \mathrm{X}-1$ is only significantly detected by Swift-XRT in the 2013 observation. Therefore for this observation, we extracted the data as described earlier, and fitted the $3-8 \mathrm{keV}$ spectrum using a simple power-law model absorbed by the Galactic column. For the other observations where NGC $5643 \mathrm{X}-1$ is not detected, 
Table 3

X-Ray Spectral Fitting Results for NGC 5643 X-1

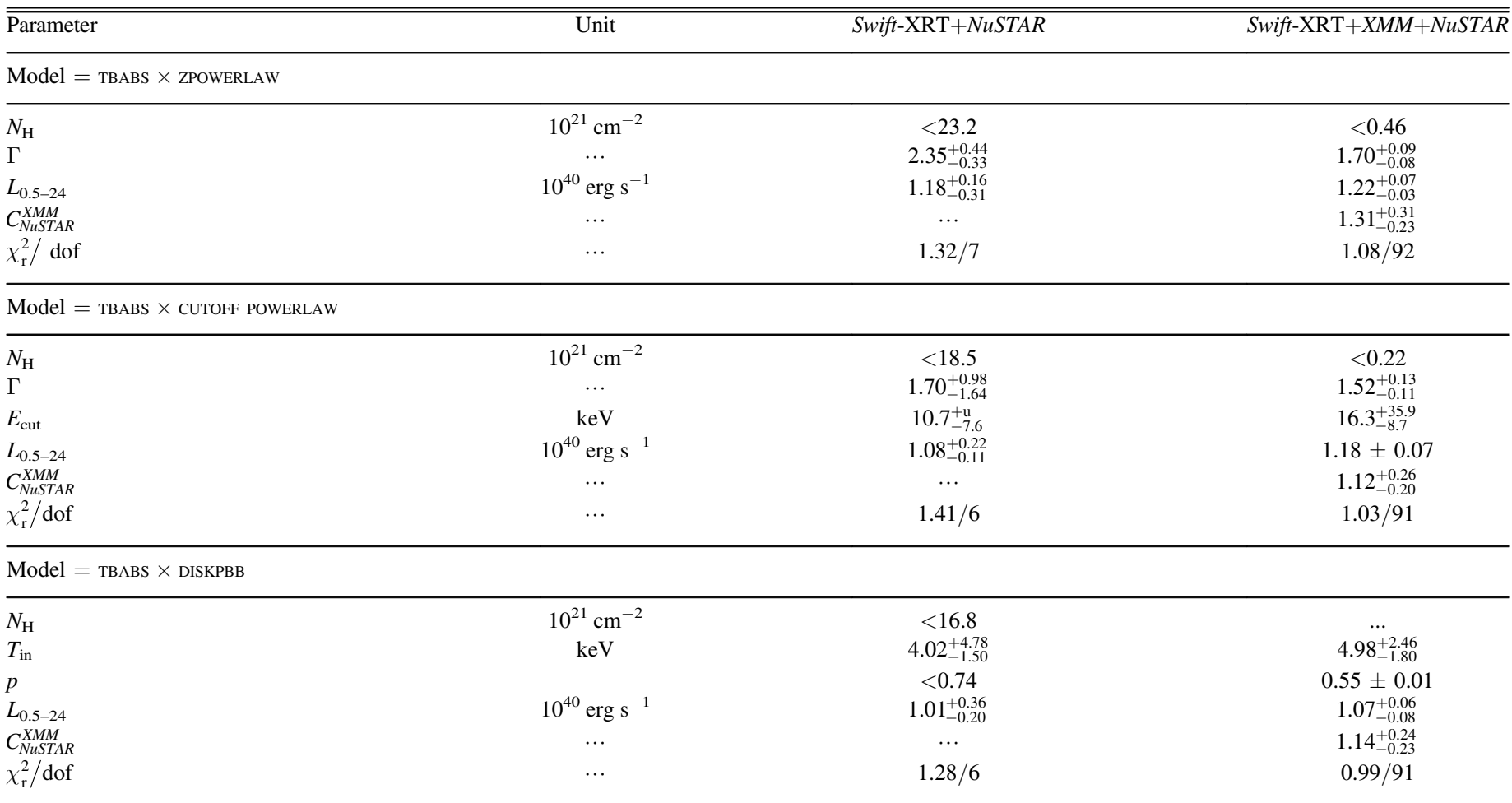

Note. ${ }^{\text {u}}$ Unconstrained.
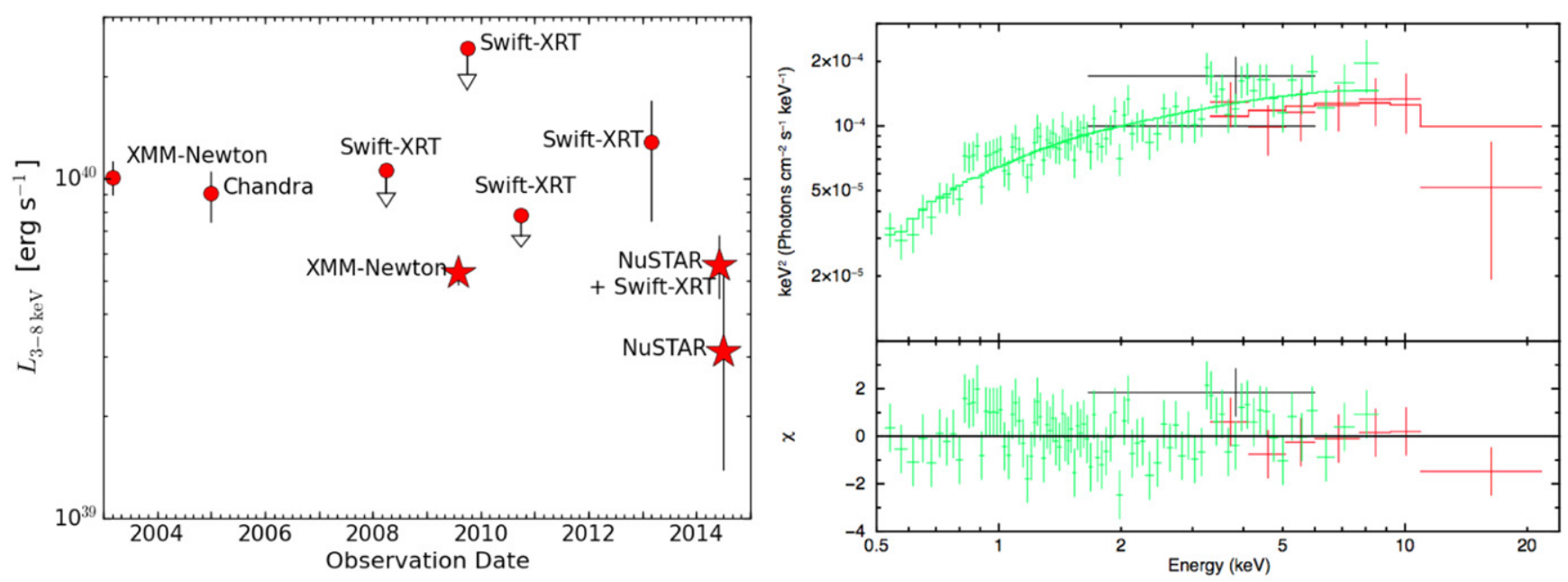

Figure 4. Left: X-ray luminosity variations of NGC $5643 \mathrm{X}-1$ in the 3-8 keV band observed between 2003 and 2014 by XMM-Newton, Chandra, Swift-XRT, and NuSTAR. Observations marked with stars are those that we used to fit the broadband spectrum of the source. Right: best-fit DISKPBB model to the combined Swift$\mathrm{XRT}+X M M+N u S T A R$ spectra of NGC $5643 \mathrm{X}-1$. The top panel shows the unfolded model in $E F_{E}$ units, while the bottom panel shows the fit residuals in terms of sigma with error bars of size one. The data have been rebinned to a minimum of $3 \sigma$ significance with a maximum of 50 bins for visual clarity. Color scheme: red (NuSTAR), black (Swift-XRT), green (XMM-Newton MOS).

we provided the upper limit luminosities at $90 \%$ confidence, estimated using aperture photometry assuming $\Gamma=2$.

We compare the $3-8 \mathrm{keV}$ luminosities between all the observations described above, as well as the NuSTAR observations at each epoch in Figure 4 to show the long term luminosity variability of NGC $5643 \mathrm{X}-1$. Note that the photon indices were left free to vary in all spectral fittings. In general, the plot shows that NGC $5643 \mathrm{X}-1$ can vary by a factor of $\sim 2-3$ (up to $\sim 5$ ) between epochs. This is broadly similar to the level of variability observed in other ULXs (e.g., Kong et al. 2010; Sutton et al. 2013; Walton et al. 2013). These results, combined with the parameters inferred by the DISKPBB 
model and evidence of an energy cutoff at $E \sim 10 \mathrm{keV}$, as well as the lack of a counterpart at other wavelengths (G04), further support the ULX classification of NGC 5643 X-1.

\section{DISCUSSION}

In Section 3, we first investigated NuSTAR observations of the CTAGN candidate in NGC 5643. We combined our new data with archival Chandra, XMM-Newton, and Swift-BAT data and performed a broadband $(\sim 0.5-100 \mathrm{keV})$ spectral analysis of the AGN. On the basis of three different reflection/ obscuration models, we found that the AGN is consistent with being CT with a column density of $N_{\mathrm{H}}(\mathrm{los}) \gtrsim 5 \times 10^{24} \mathrm{~cm}^{-2}$. The absorption-corrected 2-10 keV luminosity obtained from the models range between $L_{2-10}=(0.8-1.7) \times 10^{42} \mathrm{erg} \mathrm{s}^{-1}$, although we note that this could be a factor of a few lower/ higher due to the fact that the spectra are dominated by reflection, with negligible contribution from the direct emission, causing large uncertainties in the measurements of the intrinsic emission from the AGN. The intrinsic luminosity inferred is at the lower end of the luminosity range of the local bona fide CTAGN population (see Figure 4 in Gandhi et al. 2014). Despite the presence of a nearby ULX with a 2-10 keV luminosity comparable to the observed luminosity of the AGN itself (G04), the spectrum of the ULX drops off at $E$ $\sim 10 \mathrm{keV}$, and the overall emission above this energy is dominated by the AGN.

This paper provides the first intrinsic X-ray luminosity measurements for the AGN in NGC 5643 using CT solutions. To provide a basic test of the intrinsic luminosity estimate from our X-ray spectral fitting, we can compare the luminosity obtained to predictions from multiwavelength indicators. The obscuring torus absorbs disk radiation from the nucleus, but then re-emits it in the MIR. We can therefore use the intrinsic MIR:X-ray luminosity relationship found by Gandhi et al. (2009) based upon high angular resolution MIR $12 \mu \mathrm{m}$ observations to estimate the intrinsic X-ray luminosity of the AGN. The advantage of such observations is that because of their high angular resolution $(\sim 0$ ". 4$)$, they provide MIR fluxes that are intrinsic to the nucleus, minimizing contamination from the host galaxy. The $12 \mu \mathrm{m}$ nuclear luminosity for NGC 5643, measured by VISIR and T-ReCS mounted on the Very Large Telescope and the Gemini South telescope, respectively, is $L_{12 \mu \mathrm{m}}=(1.5 \pm 0.4) \times 10^{42} \mathrm{erg} \mathrm{s}^{-1}$ (Asmus et al. 2014). Using the Gandhi et al. (2009) relationship, this corresponds to an X-ray luminosity of $L_{2-10}=(0.6-2.5) \times 10^{42} \mathrm{erg} \mathrm{s}^{-1}$, which agrees well with the $2-10 \mathrm{keV}$ intrinsic luminosity inferred from our broadband X-ray spectral modeling. ${ }^{33}$

We also compared our intrinsic $2-10 \mathrm{keV}$ luminosity with that predicted from the optical [O III] $\lambda 5007 \AA$ emission line luminosity. [O III] $\lambda 5007 \AA$ in AGNs are mostly produced in the NLR by photoionizing radiation from the central source. Although this line is not affected by the torus obscuration, it can still suffer from obscuration by the host galaxy, which can be corrected for using the Balmer decrement. We obtained our corrected [O III] $\lambda 5007 \AA$ luminosity from Bassani et al. (1999), $L_{[\mathrm{O} \mathrm{III}]}=(1.6 \pm 0.2) \times 10^{41} \mathrm{erg} \mathrm{s}^{-1}$. Using the $L_{2-10}: L_{[\mathrm{O} \text { III] }}$ relationship from Panessa et al. (2006), we infer an X-ray luminosity of $L_{2-10}=(2.1-33.9) \times 10^{42} \mathrm{erg} \mathrm{s}^{-1}$ (see footnote 33 ). The lower end of this luminosity range is consistent with the luminosity measured from our X-ray spectral fitting. This is

\footnotetext{
33 luminosity range accounts for the mean scatter of the correlation.
}

supported by the MIR [O IV] $\lambda 25.89 \mu \mathrm{m}$ emission line, $L_{\text {[OIv }]}=(2.7 \pm 0.1) \times 10^{40} \mathrm{erg} \mathrm{s}^{-1}$ (Goulding \& Alexander 2009). Using $L_{2-10}: L_{\text {[OIv] }}$ relationship defined by Goulding et al. (2010) ${ }^{34}$, we determine an X-ray luminosity of $L_{2-10}=(0.9-5.5) \times 10^{42} \mathrm{erg} \mathrm{s}^{-1}$ (see footnote 33$)$. This luminosity agrees very well with that measured by our bestfit models.

With our updated intrinsic X-ray luminosity, we can estimate the Eddington ratio of the AGN. Using $M_{\mathrm{BH}}$ estimated from the $M_{\mathrm{BH}}-\sigma_{*}$ relation, $M_{\mathrm{BH}}=10^{6.4} M_{\odot}$ (Goulding et al. 2010; see also footnote 26), combined with the range of intrinsic X-ray power that we measured from the best-fit models, we infer that the AGN is accreting matter at about 5\%-10\% of the Eddington rate (see footnote 34 ). However, we note that this value is subject to large errors (factor of a few) due to the highly uncertain quantities involved in the calculation (Brandt \& Alexander 2015).

In the past few years, much evidence for the presence of distant cold reflecting regions has been found in nearby CTAGNs (e.g., Marinucci et al. 2013; Arévalo et al. 2014; Bauer et al. 2014). To accurately characterize the broadband spectra of these AGNs, multiple reflector components are required to model each of these reflecting regions. We investigated whether this could be the case for NGC 5643 by looking at the Chandra image of the AGN in the 6-7.5 keV band ( $\sim 30$ counts). This is shown in the top left panel of Figure 5. At first glance, the image appears to show that the iron emission region is extended well beyond the parsec-scale torus, suggesting the presence of distant cold reflecting regions. However, this appearance could also be due to an elongated PSF as a result of the position of the target on the detector, as well as the energy range used to produce the source image. We therefore used the MARX simulator ${ }^{35}$ (v4.4) to produce the expected PSF with a much higher count than the source counts ( $~ 100,000$ counts) at the position of the AGN, and at the mean energy range that we are interested in $(E=6.75 \mathrm{keV})$. This is shown in the top right panel of Figure 5. We then plotted 1D radial profiles of the target and the simulated PSF along the semimajor axis of the simulated PSF determined using the WAVDETECT tool within CIAO, and performed a KolmogorovSmirnov (KS) test between the two distributions (bottom panel of Figure 5). We found that the KS test probability is $P_{\mathrm{KS}}=0.983$, indicating that the iron emission region of the target is consistent with the simulated PSF, and therefore is not extended (see similar finding for NGC 3393 in Koss et al. 2015). A deeper (scheduled) Chandra observation could help to confirm this.

The CTAGN in NGC 5643 is located in a face-on grand design spiral galaxy with an undisturbed disk (see Figure 6). The emission lines from the AGN are not obscured/ extinguished by the host galaxy material (Goulding \& Alexander 2009), indicating that any phenomenon that might contribute to the CT obscuration in this source is therefore likely to occur very close to the nucleus. Indeed, there have been indications that the emission at the west side of the nucleus is obscured by a warped disk or the presence of a dust lane (e.g., Simpson et al. 1997; Fischer et al. 2013; Davies

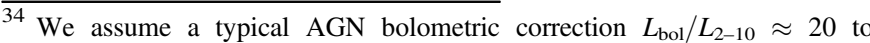
calculate the bolometric luminosity and hence the mass accretion rate of the AGN (e.g., Elvis et al. 1994; Vasudevan et al. 2010).

35 The MARX simulation documentation is available at http://space.mit.edu/ ASC/MARX/.
} 

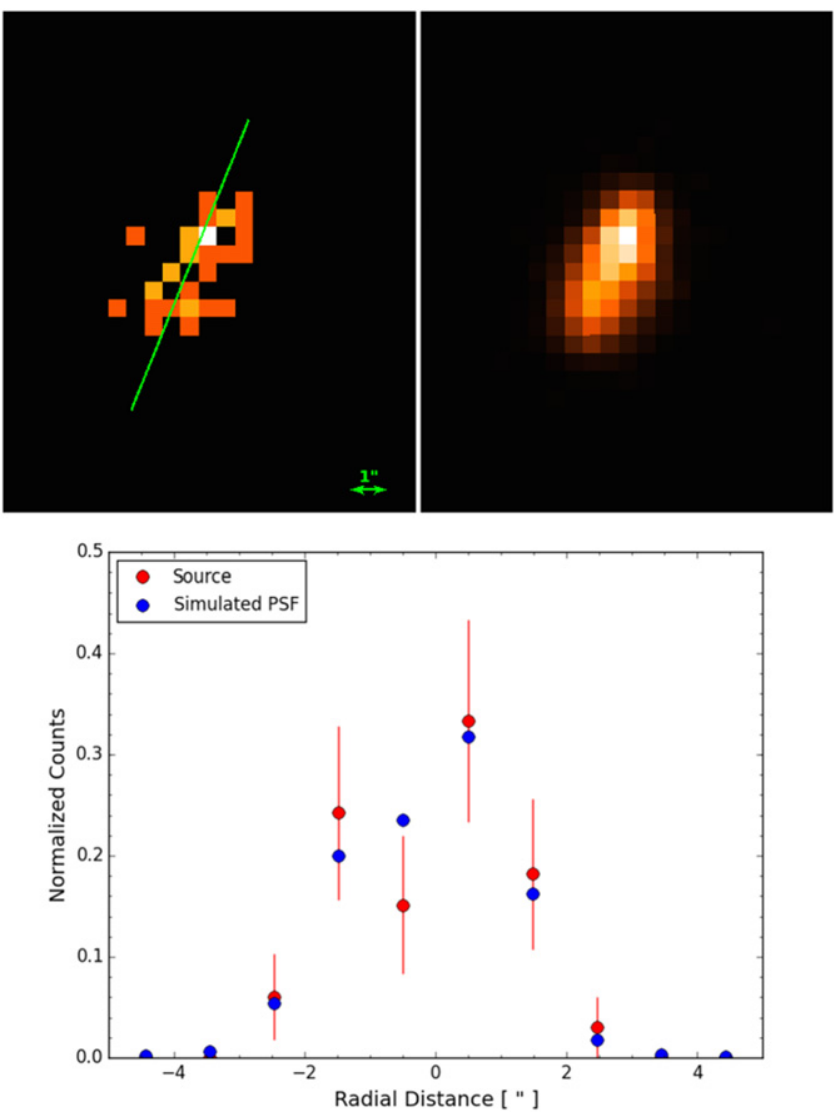

Figure 5. Top: Chandra 6-7.5 keV images of the AGN (left) and the simulated PSF (right). The green line shows the direction of the semimajor axis of the simulated PSF that was used to produce the 1D radial profiles for both the source and the simulated PSF. Bottom: the 1D radial profiles of the source (red) and the simulated PSF (blue) normalized to their respective total counts. The error bars indicate the $1-\sigma$ Poisson errors for the data.

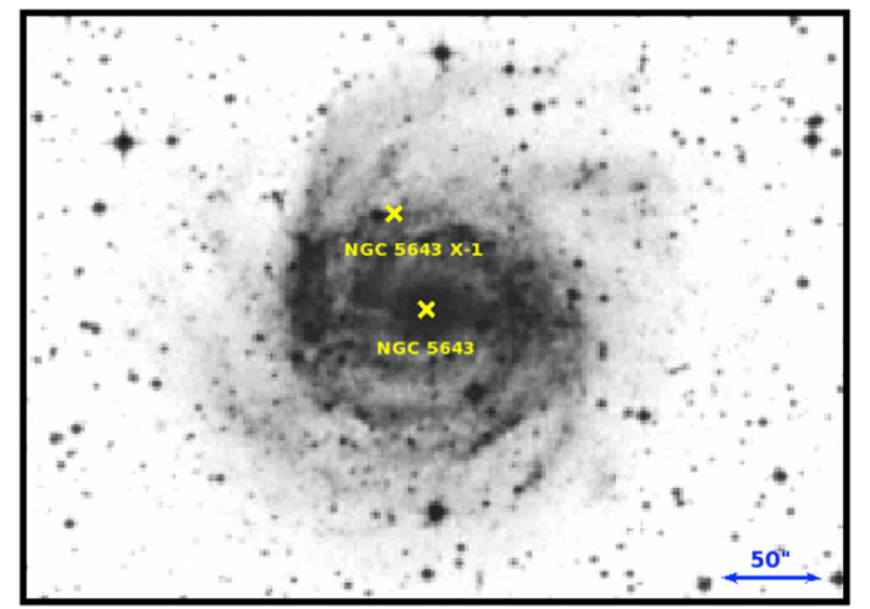

Figure 6. Optical image of NGC 5643 retrieved from the ESO Digital Sky Survey image server. The Chandra position of the AGN (NGC 5643) and the ULX candidate (NGC $5643 \mathrm{X}-1$ ) are both labeled and marked with an " $\times$." North is up and east is to the left in the image.

et al. 2014). The torus itself could be the inner structure of this disk. Because of the more direct and "cleaner" view of the central engine in NGC 5643 as compared to some other very nearby bona fide CTAGNs such as NGC 1068, NGC 4945, and Circinus, this makes NGC 5643 a good candidate for more detailed studies of the CT obscurer in the nuclear region.

\section{SUMMARY}

We observed the AGN and ULX candidate in NGC 5643 using NUSTAR on two occasions conducted about a month apart. A summary of our main results is as follows.

1. Using the combined data from NuSTAR, Chandra, XMMNewton, and Swift-BAT, we have extended the broadband spectral analysis of the CTAGN candidate in NGC 5643 to high energies $(\sim 0.5-100 \mathrm{keV})$. Using physically motivated toroidal obscuration models, we showed that the source is indeed CT, with a column density of $N_{\mathrm{H}}(\mathrm{los})$ $\gtrsim 5 \times 10^{24} \mathrm{~cm}^{-2}$.

2. The absorption-corrected $2-10 \mathrm{keV}$ luminosity measured by these models is $L_{2-10}$, int $=(0.8-1.7) \times 10^{42} \mathrm{erg} \mathrm{s}^{-1}$, consistent with that predicted from multiwavelength intrinsic luminosity indicators. The luminosity inferred is at the lower end of the luminosity range of the local CTAGN population.

3. The NUSTAR spectra of the off-nuclear source, NGC $5643 \mathrm{X}-1$, shows evidence for a spectral cutoff at $E \sim$ $10 \mathrm{keV}$, similar to that observed in other ULXs observed by NUSTAR. Combining this information with the evidence for X-ray luminosity variations observed between different observations, along with the absence of unambiguous counterparts at other wavelengths, we concluded that the source is consistent with being a ULX. Future simultaneous low- and high energy X-ray observations of this field are needed in order to provide higher quality data to confirm the spectral cutoff that we observed and to better characterize the broadband spectrum of the source.

The authors thank the anonymous referee for useful comments that have helped to improve the paper. We thank Chris Done for some discussions on the residuals around the iron line complex. A.A. thanks Wasutep Luangtip for useful discussion on NGC $5643 \mathrm{X}-1$ and help with the MARX simulation. We also thank Neil Gehrels and the Swift team for the simultaneous Swift-XRT observation. We acknowledge financial support from Majlis Amanah Rakyat (MARA) Malaysia (A.A.), the Science and Technology Facilities Council (STFC) grants ST/J003697/1 (P.G.), ST/I0015731/ 1 (D.M.A. and A.D.M.), and ST/K501979/1 (G.B.L.), the Leverhulme Trust (D.M.A.), NASA Headquarters under the NASA Earth and Space Science Fellowship Program grant NNX14AQ07H (M.B.), CONICYT-Chile grants Basal-CATA PFB-06/2007 (F.E.B.), FONDECYT 1141218 (F.E.B.), and "EMBIGGEN" Anillo ACT1101 (F.E.B.); the Ministry of Economy, Development, and Tourism's Millennium Science Initiative through grant IC120009, awarded to The Millennium Institute of Astrophysics, MAS (F.E.B.).

NUSTAR is a project led by the California Institute of Technology (Caltech), managed by the Jet Propulsion Laboratory (JPL), and funded by the National Aeronautics and Space Administration (NASA). We thank the NuSTAR Operations, Software and Calibrations teams for support with these observations. This research has made use of the NuSTAR Data Analysis Software (NUSTARDAS) jointly developed by the ASI Science Data Center (ASDC, Italy) and the California Institute of Technology (USA), and the XRT Data Analysis Software (XRT-DAS). This research also made use of the data obtained through the High Energy Astrophysics Science Archive 
Research Center (HEASARC) Online Service, provided by the NASA/Goddard Space Flight Center, and the NASA/IPAC extragalactic Database (NED) operated by JPL, Caltech under contract with NASA.

Facilities: NuSTAR, XMM, CXO, Swift.

\section{REFERENCES}

Abramowicz, M. A., Czerny, B., Lasota, J. P., \& Szuszkiewicz, E. 1988, ApJ, 332,646

Akylas, A., Georgakakis, A., Georgantopoulos, I., Brightman, M., \& Nandra, K. 2012, A\&A, 546, A98

Alexander, D. M., \& Hickox, R. C. 2012, NewAR, 56, 93

Arévalo, P., Bauer, F. E., Puccetti, S., et al. 2014, ApJ, 791, 81

Asmus, D., Hönig, S. F., Gandhi, P., Smette, A., \& Duschl, W. J. 2014 , MNRAS, 439, 1648

Bachetti, M., Rana, V., Walton, D. J., et al. 2013, ApJ, 778, 163

Baloković, M., Comastri, A., Harrison, F. A., et al. 2014, ApJ, 794, 111

Barthelmy, S. D., Barbier, L. M., Cummings, J. R., et al. 2005, SSRv, 120, 143

Bassani, L., Dadina, M., Maiolino, R., et al. 1999, ApJS, 121, 473

Bauer, F. E., Arevalo, P., Walton, D. J., et al. 2014, arXiv:1411.0670

Baumgartner, W. H., Tueller, J., Markwardt, C. B., et al. 2013, ApJS, 207, 19

Bianchi, S., Guainazzi, M., \& Chiaberge, M. 2006, A\&A, 448, 499

Brandt, W. N., \& Alexander, D. M. 2015, A\&ARv, 23, 1

Brightman, M., \& Nandra, K. 2011, MNRAS, 413, 1206

Burlon, D., Ajello, M., Greiner, J., et al. 2011, ApJ, 728, 58

Burrows, D. N., Hill, J. E., Nousek, J. A., et al. 2005, SSRv, 120, 165

Cash, W. 1979, ApJ, 228, 939

Cid Fernandes, R., Heckman, T., Schmitt, H., González Delgado, R. M., \& Storchi-Bergmann, T. 2001, ApJ, 558, 81

Davies, R. I., Maciejewski, W., Hicks, E. K. S., et al. 2014, ApJ, 792, 101

Della Ceca, R., Severgnini, P., Caccianiga, A., et al. 2008, MmSAI, 79, 65

Draper, A. R., \& Ballantyne, D. R. 2010, ApJL, 715, L99

Elvis, M., Wilkes, B. J., McDowell, J. C., et al. 1994, ApJS, 95, 1

Fabian, A. C. 1999, MNRAS, 308, L39

Fischer, T. C., Crenshaw, D. M., Kraemer, S. B., \& Schmitt, H. R. 2013, ApJS, 209, 1

Gandhi, P., Horst, H., Smette, A., et al. 2009, A\&A, 502, 457

Gandhi, P., Lansbury, G. B., Alexander, D. M., et al. 2014, ApJ, 792, 117

Genzel, R., Lutz, D., Sturm, E., et al. 1998, ApJ, 498, 579

Gilli, R., Comastri, A., \& Hasinger, G. 2007, A\&A, 463, 79

Goulding, A. D., \& Alexander, D. M. 2009, MNRAS, 398, 1165

Goulding, A. D., Alexander, D. M., Bauer, F. E., et al. 2012, ApJ, 755, 5

Goulding, A. D., Alexander, D. M., Lehmer, B. D., \& Mullaney, J. R. 2010, MNRAS, 406, 597

Goulding, A. D., Alexander, D. M., Mullaney, J. R., et al. 2011, MNRAS, 411, 1231

Greenhill, L. J., Kondratko, P. T., Lovell, J. E. J., et al. 2003, ApJL, 582, L11

Gu, Q., Melnick, J., Cid Fernandes, R., et al. 2006, MNRAS, 366, 480

Guainazzi, M., Rodriguez-Pascual, P., Fabian, A. C., Iwasawa, K., \& Matt, G. 2004, MNRAS, 355, 297

Harrison, F. A., Craig, W. W., Christensen, F. E., et al. 2013, ApJ, 770, 103

Iwasawa, K., Fabian, A. C., \& Matt, G. 1997, MNRAS, 289, 443
Jonker, P. G., Heida, M., Torres, M. A. P., et al. 2012, ApJ, 758, 28

Kalberla, P. M. W., Burton, W. B., Hartmann, D., et al. 2005, A\&A, 440, 775

Kong, A. K. H., Yang, Y. J., Yen, T.-C., Feng, H., \& Kaaret, P. 2010, ApJ, 722,1816

Koss, M. J., Romero-Canizales, C., Baronchelli, L., et al. 2015, arXiv: 1505.03524

Lansbury, G. B., Alexander, D. M., Del Moro, A., et al. 2014, ApJ, 785, 17

Liu, Y., \& Li, X. 2015, MNRAS, 448, L53

Luo, B., Brandt, W. N., Alexander, D. M., et al. 2013, ApJ, 772, 153

Madsen, K. K., Harrison, F. A., Markwardt, C., et al. 2015, arXiv:1504.01672

Magdziarz, P., \& Zdziarski, A. A. 1995, MNRAS, 273, 837

Maiolino, R., Salvati, M., Bassani, L., et al. 1998, A\&A, 338, 781

Marinucci, A., Miniutti, G., Bianchi, S., Matt, G., \& Risaliti, G. 2013, MNRAS, 436, 2500

Matt, G., Bianchi, S., Marinucci, A., et al. 2013, A\&A, 556, A91

Matt, G., Fabian, A. C., Guainazzi, M., et al. 2000, MNRAS, 318, 173

Matt, G., Guainazzi, M., Frontera, F., et al. 1997, A\&A, 325, L13

McConnell, N. J., \& Ma, C.-P. 2013, ApJ, 764, 184

Miller, J. M., Raymond, J., Fabian, A. C., et al. 2004, ApJ, 601, 450

Mineshige, S., Hirano, A., Kitamoto, S., Yamada, T. T., \& Fukue, J. 1994, ApJ, 426, 308

Morris, S., Ward, M., Whittle, M., Wilson, A. S., \& Taylor, K. 1985, MNRAS, 216, 193

Mould, J. R., Huchra, J. P., Freedman, W. L., et al. 2000, ApJ, 529, 786

Murphy, K. D., \& Yaqoob, T. 2009, MNRAS, 397, 1549

Orosz, J. A. 2003, in IAU Symp. 212, A Massive Star Odyssey: From Main Sequence to Supernova, ed. K. van der Hucht, A. Herrero \& C. Esteban (San Francisco, CA), 365

Panessa, F., Bassani, L., Cappi, M., et al. 2006, A\&A, 455, 173

Phillips, M. M., Charles, P. A., \& Baldwin, J. A. 1983, ApJ, 266, 485

Poutanen, J., Lipunova, G., Fabrika, S., Butkevich, A. G., \& Abolmasov, P. 2007, MNRAS, 377, 1187

Puccetti, S., Comastri, A., Fiore, F., et al. 2014, ApJ, 793, 26

Rana, V., Harrison, F. A., Bachetti, M., et al. 2015, ApJ, 799, 121

Risaliti, G., Maiolino, R., \& Salvati, M. 1999, ApJ, 522, 157

Sanders, D. B., Mazzarella, J. M., Kim, D.-C., Surace, J. A., \& Soifer, B. T. 2003, AJ, 126, 1607

Shakura, N. I., \& Sunyaev, R. A. 1973, A\&A, 24, 337

Simpson, C., Wilson, A. S., Bower, G., et al. 1997, ApJ, 474, 121

Stern, D., Lansbury, G. B., Assef, R. J., et al. 2014, ApJ, 794, 102

Strohmayer, T. E. 2009, ApJL, 706, L210

Sutton, A. D., Roberts, T. P., Gladstone, J. C., et al. 2013, MNRAS, 434, 1702

Sutton, A. D., Roberts, T. P., Walton, D. J., Gladstone, J. C., \& Scott, A. E. 2012, MNRAS, 423, 1154

Swartz, D. A., Soria, R., Tennant, A. F., \& Yukita, M. 2011, ApJ, 741, 49

Treister, E., Urry, C. M., \& Virani, S. 2009, ApJ, 696, 110

Ueda, Y., Akiyama, M., Hasinger, G., Miyaji, T., \& Watson, M. G. 2014, ApJ, 786, 104

Vasudevan, R. V., Fabian, A. C., Gandhi, P., Winter, L. M., \& Mushotzky, R. F. 2010, MNRAS, 402, 1081

Walton, D. J., Fuerst, F., Harrison, F., et al. 2013, ApJ, 779, 148

Walton, D. J., Gladstone, J. C., Roberts, T. P., et al. 2011a, MNRAS, 414, 1011

Walton, D. J., Harrison, F. A., Grefenstette, B. W., et al. 2014, ApJ, 793, 21

Walton, D. J., Roberts, T. P., Mateos, S., \& Heard, V. 2011b, MNRAS, 416, 1844 